\title{
Immunoinformatics Prediction of Epitope Based Peptide Vaccine Against Madurella mycetomatis Translationally Controlled Tumor Protein
}

\author{
Samira Munir Bolis ${ }^{1,2}$, Walaa Abdullah Omer ${ }^{1,2^{*}}$, Mohamed Anwar Abdelhamed ${ }^{2}$, Masajed \\ Abdelmagid Shambal ${ }^{2}$, Esameldeen Ahmed Adam ${ }^{2}$, Mohammed Abaker Abass ${ }^{2,3}$, Wiaam \\ Abdelwahab Abdalla ${ }^{2}$, Suzan Hashim Is-haq ${ }^{4,2}$, Aisha Abubakralsiddig Abdalla ${ }^{2}$, Abeer Algaali \\ Zeinalabedeen ${ }^{2}$, Omar Hashim Ahmed ${ }^{5 *}$, Mohamed A Hassan ${ }^{2}$. \\ 1. Department of Medical Micrbiology, Faculty of Medical laboratories Sciences, University of Khartoum, Sudan. \\ 2. Department of Biotechnology, Africa City of Technology, Sudan. \\ 3. Department of Medical Micrbiology, Faculty of Medical laboratories Sciences, Sudan International University, \\ Sudan. \\ 4. Department of clinical chemistry, Faculty of Medical laboratories Sciences, University of Science and \\ Technology, Sudan. \\ 5. Department of Pharmacology, Faculty of Pharmacy, University of Gazira, Sudan. \\ Corresponding Authors: \\ * Omar Hashim Ahmed, Department of Pharmacology, Faculty of Pharmacy, University of Gazira, Sudan, Mob: \\ +249912683595, email Ohashim221@gmail.com. \\ *Walaa Abdullah Omer, Department of Medical Micrbiology, Faculty of Medical laboratories Sciences, University \\ of Khartoum, Sudan, Mob: +24991220555, email walloya2@hotmail.com.
}

\begin{abstract}
Background: Madurella. mycetomatis is most common causative agent of mycetoma in Sudan and worldwide. No vaccines are available till now so design of effective vaccine is essential as protection tool. Peptide vaccine can overcome the common side effects of the conventional vaccines. The aim of this study was to design peptide based vaccine for M.Mycetomatis Translationally Controlled Tumor Protein (TCTP) using immunoinformatics tools.
\end{abstract}

Materials and methods: TCTP sequences were retrieved from NCBI and then processed using BioEdit program to determine conserved regions and different immunoinformatics tools from IEDB. Population coverage analysis was performed for the most promising epitopes. Homology modelling was performed to show their structural positions in TCTP. Protein analysis was done using Expasy (ProtParamsotware).

Results and conclusion: Four epitopes passed the Bepipred, Emini, Kolaskar and Tongaonkar tools. 111 epitopes were predicted to interact with MHCI alleles with IC50<500 nM, three of them were most promising. 274 predicted epitopes were interacted with MHCII alleles with IC50 $<100 \mathrm{nM}$, four of them were most promising. The epitope (YMKSVKKAL) was the most promising one concerning its binding with MHCI alleles, while (FRLQSTSFD) was the most promising for MHC II. The epitope (YLKAYMKSV) is shared betweenMHC I and II. For the population coverage of M. Mycetomatis TCTP vaccine Sudan $(90.39 \%)$ had the highest percentage for MHC I. This is the first computational vaccinology study conducted in mycetoma caused by M. Mycetomatis using TCTP.

\section{Keywords}

Madurella.Mycetomatis, mycetoma, Sudan, worldwide, peptide vaccine, Translationally Controlled Tumor Protein(TCTP), immunoinformatics tools. 


\section{Introduction}

Mycetoma is a granulomatous subcutaneous chronic progressive infection. It is characterized by a painless swelling, development of draining sinuses that discharge different colors of pus and grains (1-3).Mycetoma is subdivided into actinomycetoma and eumycetoma $(1,4,5)$. It is distributed worldwide, but it is endemic in tropical and subtropical regions (6-8). These region sknown as the "Mycetoma belt" including (Sudan, Somalia, Yemen, Senegal, India, Mexico, Venezuela, Colombia and Argentina) while the most reported cases were from Sudan and Mexico, Sudan being the most endemic country (9) $(10,11)$. Mycetoma can be found in areas geographically close proximity to Tropic of Cancer (1). It is categorized as one of the neglected tropical diseases at $2013(6,12)$. Accurate data on its incidence and prevalence are not available (12). The most common causative organism of mycetoma in Sudan and worldwide is Madurella mycetomatis (13-15). Mycetoma is still widely distributed in Sudan with no effective treatment, no vaccines are available till now hence the design of an effective peptide vaccine is essential as protection tool against the disease (12). This study aimed to predict an insilico peptide based vaccine for mycetoma caused by $M$. mycetomatis using immunoinformatics.

The defense mechanisms against fungi usually range from early non-specific immune response to activation of specific adaptive immune responses by the production of cytokines of $\mathrm{T}$ helper-1 and $\mathrm{T}$ helper-2. Cell-mediated immunity can play a role in the pathogenesis of eumycetoma (16). The first well-characterized immunogenic antigen is Translationally Controlled Tumor Protein (TCTP).Significant IgG and IgM immune responses against this protein were determined. The level of antibodies is directly proportional to lesion size and duration of the disease (17). Different laboratory-based diagnostic techniques and tools were developed to determine and identify the causative agents of mycetoma (18). Early detection and treatment are important to reduce morbidity and improve treatment outcomes $(5,16)$. Antifungals are used for the treatment of eumycetoma, amputations and recurrences are common $(4,8)$. Recently understanding of complex interactions between fungi and host led to exploration and design of novel vaccines (19). The first genome sequence for this strain in Sudan was presented in 2016, better therapies for mycetoma will be developed using this sequence (13).TCTP is abundantly expressed in wide range of eukaryotic organisms located in the cytoplasm and the nucleus $(20,21)$. It is highly conserved protein and its expression is associated with malignancy and chemoresistance. TCTP 
plays important role in physiological events, such as immune responses, cell proliferation and cell death (20). TCTP is simultaneously the first identified monomolecular vaccine candidate for M.mycetomatis (17). Next to the TCTP, two antigenic proteins of M.mycetomatis were discovored, the glycolytic enzymes Fructose Biphosphate Aldolase (FBA) and Pyruvate Kinase $(P K)$, these antigens might also be useful as vaccine-candidates in the prevention of mycetoma (22). The gene for this protein was found to be present in two variants in M. mycetomatis, with difference in 13\% amino acids between the two encoded proteins (17). This is the first computational vaccinology study conducted in mycetoma caused by M.mycetomatis using TCTP.

\section{Materials and methods}

\section{Protein sequence retrieval}

Nine Madurella mycetomatis TCTP sequences were retrieved from variants one and two from the National Center for Biotechnology Information (NCBI) (https://www.ncbi.nlm.nih.gov/protein/?term=mycetoma+and+TCTP) database in August 2017.

\section{Determination of conserved regions}

Multiple sequence alignment (MSA) using ClustalW as in the BioEdit program, version 7.2.5 was used to obtain conserved regions among Madurella. mycetomatis TCTP (23).

\section{B-cell epitope prediction}

The reference Madurella. mycetomatis TCTP sequence was subjected to Bepipred linear epitope, Emini surface accessibility and Kolaskar and Tongaonkar antigenicity methods in Immune Epitope Data Base (IEDB) (22-24) (http://www.iedb.org/), that predict the probability of specific regions in the protein to bind to B cell receptor, being linear, in the surface and immunogenic, respectively.

\section{Prediction of linear B-cell epitopes}

Bepipred from immune epitope database (http://tools.iedb.org/bcell/) was used as linear B-cell epitopes prediction tool from the conserved region with a default threshold value of $0.240(25)$. 


\section{Prediction of surface accessibility}

By using Emini surface accessibility prediction tool of the (IEDB) (22-24) (http://tools.iedb.org/bcell/result/). The surface accessible epitopes were predicted from the conserved region holding the default threshold value 1.000 (26).

\section{Prediction of epitopes antigenicity sites}

The kolaskar and tongaonker antigenicity method (http://tools.iedb.org/bcell/result) was used to determine the antigenic sites with a default threshold value of 1.024 (27) .

\section{MHC class I binding predictions}

Analysis of peptide binding to MHC class I molecules was assessed by the (IEDB) MHC I prediction tool at (http://tools.iedb.org/mhci/result/). Peptide complex presentations to $\mathrm{T}$ lymphocytes undergo several steps. The attachment of cleaved peptides to MHC molecules step was predicted. Prediction methods was achieved by Artificial Neural Network (ANN) method (28-32). Prior to prediction, all epitope lengths were set as 9amino acids, all conserved epitopes that bind to alleles at score less than 500 inhibitory concentration (IC50) were selected for further analysis (32).

\section{MHC class II binding predictions}

Analysis of peptide binding to MHC class II molecules was assessed by the IEDB MHC II prediction tool at (http://tools.iedb.org/mhcii/result/) $(33,34)$. For MHC-II binding predication, human allele references set were used. , NN-align uses the artificial neural networks that allows for simultaneous identification of the MHC class II binding core epitopes and binding affinity from IEDB was used as prediction methods for MHC II (35). All conserved epitopes that bind to many alleles at score less than 100 half-maximal inhibitory concentration(IC50) were selected for further analysis (33). 


\section{USCF Chimera version $\mathbf{1 . 8}$}

It is a program for visualization and analysis of molecular structures and related data with highquality images and animations. It is produced by University of California, San Francisco. Fallowing step by step to generate models for Madurella mycetomatis TCTP. (Available at https://www.cgl.ucsf.edu/chimera/).

\section{Population coverage calculation}

Selected MHC-I and MHC-II interacted alleles by the IEDB population coverage calculation tool at (http://tools.iedb.org/tools/population/iedb_input) (36).

\section{Results}

\section{Protein sequence retrieval}

Retrieved TCTP sequences and their accession numbers are: ABB20812, ABB20815, ABB20814, ABB20813,ABB20811, ABB20810, ABB20809, ABB20808, ABB20807.

\section{Alignment \\ Madurella mycetomatis TCTP conserved regions are shown in (figure 1). Prediction of B-cell epitope:}

In Bepipred Linear Epitope Prediction method; the average binders score of TCTP to B cell was 0.240, with maximum score 1.974 and a minimum score -1.296 (figure 2). Seven epitopes were predicted eliciting B lymphocyte from the conserved regions and all values equal or greater than the default threshold 0.24 .

In Emini surface accessibility prediction; the average surface accessibility areas of the protein was scored as 1.000 , with a maximum of 2.963 and a minimum of 0.073 (figure 3 ). 37 epitopes were potentially in the surface by passing the default threshold 1.000 .

In Kolaskar and Tongaonkar antigenicity; the average of the antigenicity was 1.024, with a maximum of 1.188 and minimum of 0.892 (figure 4). 15 epitopes gave score above the default threshold 1.024 . 
There are four epitopes successfully passed the three tools (DEVKEFETKAQAYV, EVKEFETKAQAYV, EFETKAQAYV, ETKAQAYV) as shown in (table 1).

The positions of proposed conserved B cell epitopes in structural level of TCTP of Madurella. mycetomatis are shown in (figure 5).

\section{Prediction of cytotoxic T-lymphocyte epitopes and interaction with MHC class I:}

The reference TCTP sequence was analyzed using (IEDB) MHC-1 binding prediction tool to predict T cell epitope suggested interacting with different types of MHC Class I alleles, based on Artificial Neural Network (ANN) with half-maximal inhibitory concentration (IC50) $<500$ nm.111peptides were predicted to interact with different MHC-1alleles. The most promising epitopes and their corresponding MHC-1 alleles are shown in (Table 2). Their positions at the structural level were determined by the aid of the chimera software as shown in (Figures 6,Figures7).

\section{Prediction of The T cell epitopes and interaction with MHC class II}

Reference TCTP variant was analyzed using (IEDB) MHC-II binding prediction tool based on $\mathrm{NN}$-align with half-maximal inhibitory concentration (IC50) $<100 \mathrm{~nm}$; there were 274 predicted epitopes found to interact with MHC-II alleles.

The results of top four epitopes are listed in (Table 3). Their positions at the structural level were determined by the aid of the chimera software as shown in (Figure 8,Figure 9).

\section{Protein analysis}

M.mycetomatis TCTP protein sequence was analyzed using both Bioedit program, version 7.0.9.0 and Expasy (ProtParam software) (https://web.expasy.org/cgi-bin/protparam/protparam) softwares' tools (figure 10). Number of amino acids: 141. Molecular weight: 15794.80

Total number of negatively charged residues $(\mathrm{Asp}+\mathrm{Glu})$ : 25. Total number of positively charged residues (Arg + Lys): 17. The Atomic composition of Madurella mycetomatisTCTP is shown in (Table 4). 
Formula: C705H1095N177O224S5. Total number of atoms: 2206. The N-terminal of the sequence considered is $\mathrm{V}$ (Val). The estimated half-life is: 100 hours (mammalian reticulocytes, in vitro), $>20$ hours (yeast, in vivo) and >10 hours (Escherichia coli, in vivo).

Instability index: The instability index (II) is computed to be 29.38 (the protein is stable). Grand average of hydropathicity (GRAVY): -0.428

\section{Population coverage calculation}

All promising MHC I and MHC II binders of Madurella mycetomatis TCTP (epitopes with high binding affinity with different sets of alleles) were assessed for population coverage against the whole world and Sudan (table 5).

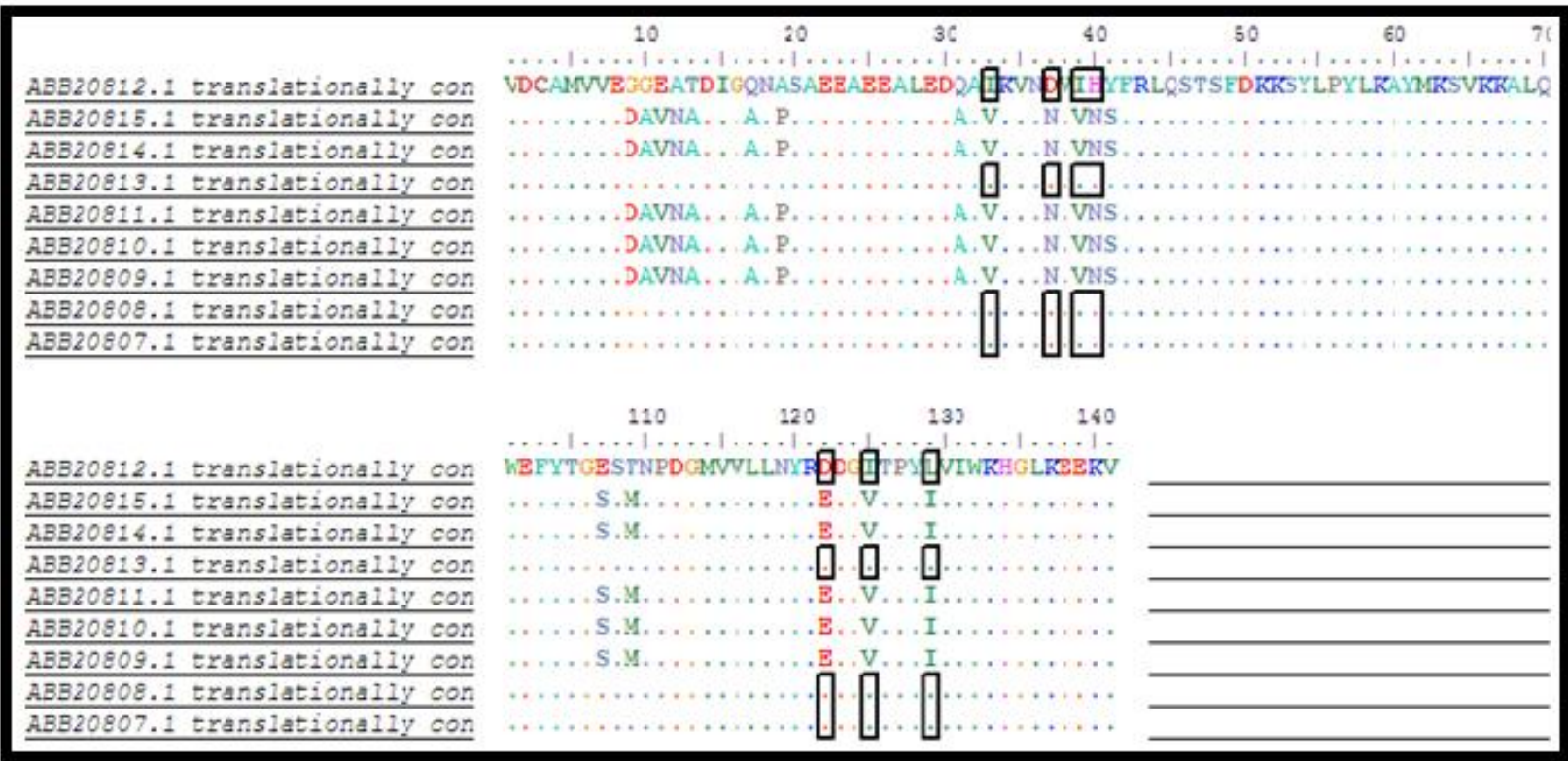

Fig 1 Multiple sequence alignment, dots show the conservancy between sequences *The alignment is done using BioEdit tool. 


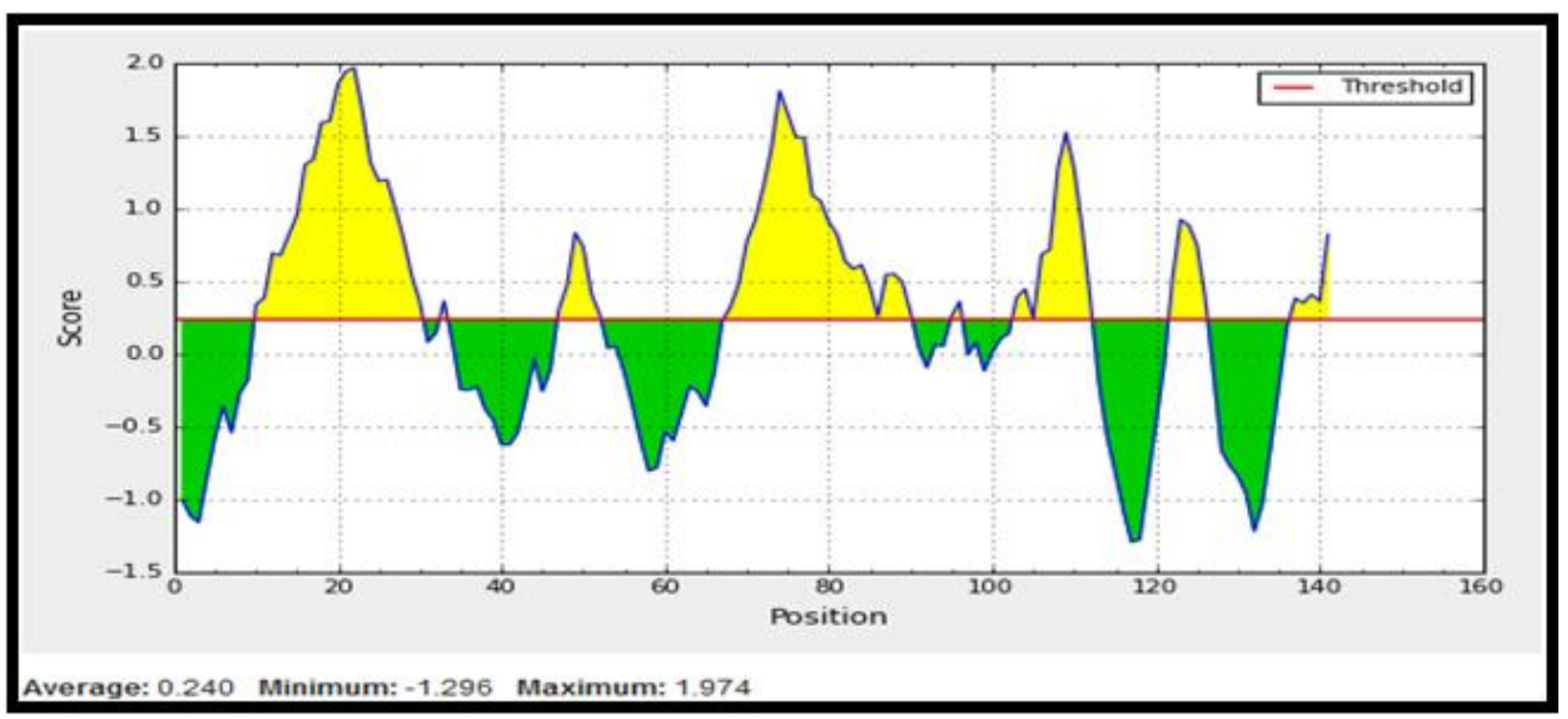

Fig 2 Bepipred linear epitope prediction.Yellow areas above threshold (red line) are proposed to be a part of B cell epitope. While green areas are not

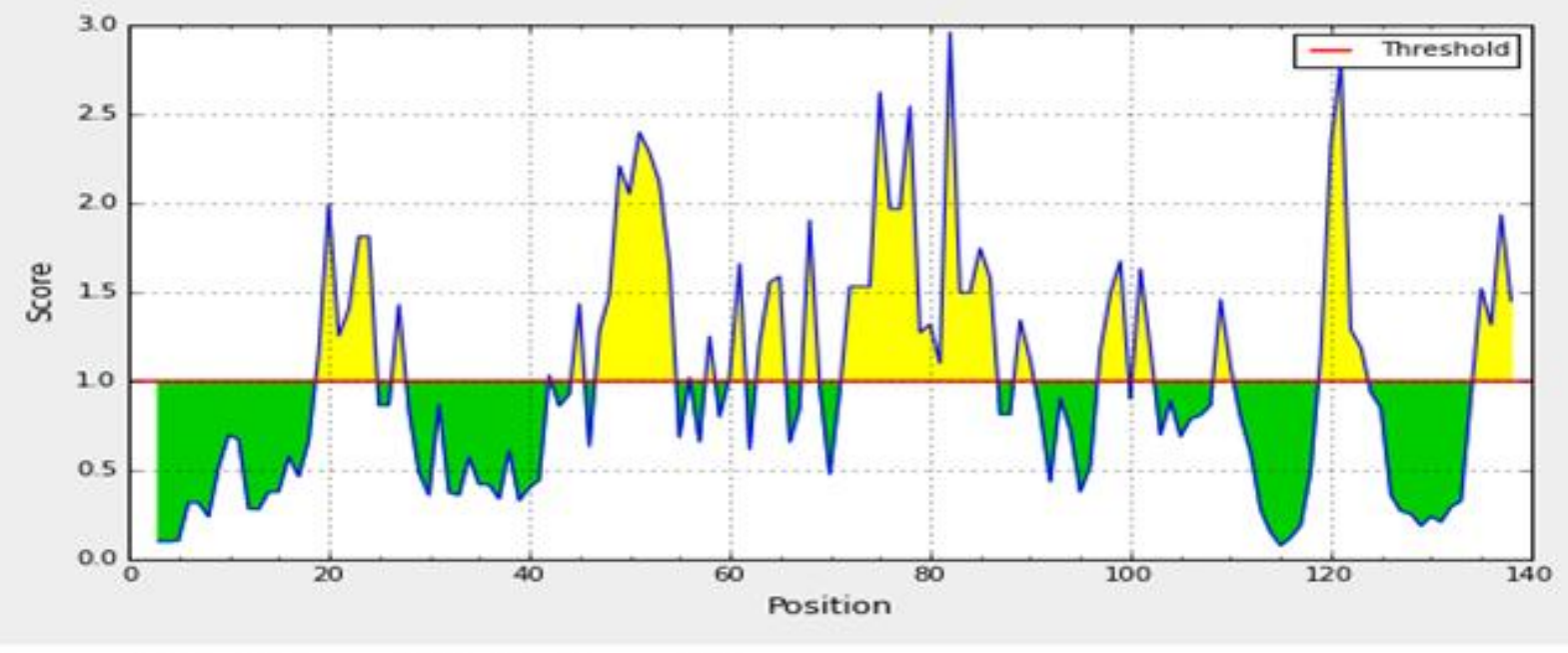

Average: 1.000 Minimum: 0.073 Maximum: 2.963

Fig 3 Emini surface accessibility prediction. Yellow areas above threshold (red line) are proposed to be a part of B cell epitope. While green areas are not. 


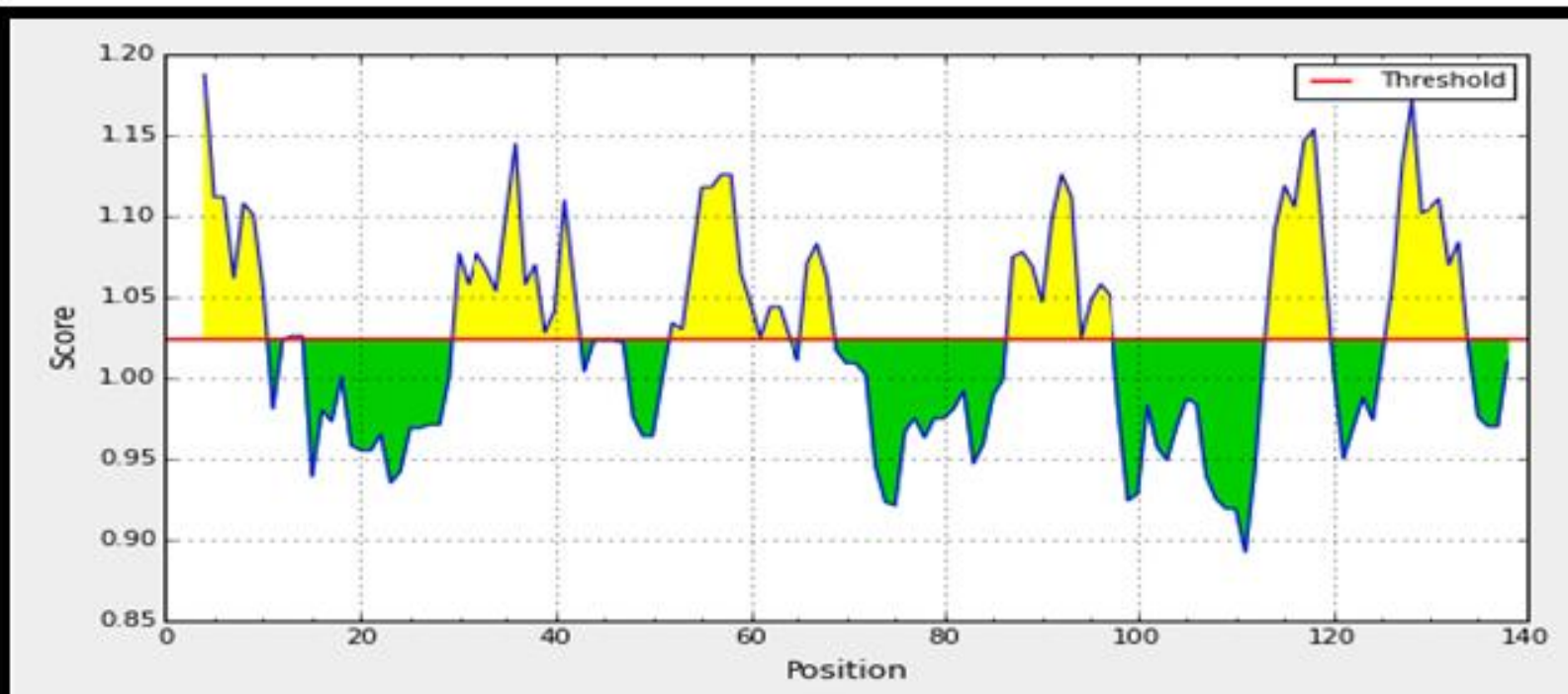

Average: 1.024 Minimum: 0.892 Maximum: 1.188

Fig 4 Kolaskar and Tongaonkar antigenicity prediction. Yellow areas above threshold (red line) are proposed to be a part of B cell epitope. While green areas are not.

Table 1: B cell epitopes prediction, Peptides in the red color have passed the three tests of the Bcell.

\begin{tabular}{cccccc}
\hline Peptide & Length & Start & End & Eminiscore & Antiegenicity score \\
\hline DIG & 3 & 14 & 16 & 0.503 & 0.964 \\
SAEEAEEALED & 11 & 20 & 30 & 1.544 & 0.961 \\
SAEEAEEALE & 10 & 20 & 29 & 1.192 & 0.971 \\
SAEEAEEAL & 9 & 20 & 28 & 0.893 & 0.984 \\
SAEEAEEA & 8 & 20 & 27 & 1.421 & 0.951 \\
SAEEAEE & 7 & 20 & 26 & 1.869 & 0.935 \\
SAEEAE & 6 & 20 & 25 & 1.404 & 0.949 \\
SAEEA & 5 & 20 & 24 & 1.046 & 0.968 \\
SAEE & 4 & 20 & 23 & 1.352 & 0.945 \\
SAE & 3 & 20 & 22 & 1.019 & 0.976 \\
TSFDKK & 6 & 47 & 52 & 2.211 & 0.956 \\
TSFDK & 5 & 47 & 51 & 1.426 & 0.962 \\
TSFD & 4 & 47 & 50 & 0.931 & 0.97 \\
TSF & 3 & 47 & 49 & 0.728 & 1.004
\end{tabular}




\begin{tabular}{|c|c|c|c|c|c|}
\hline ALQDAGKSEDEVKEFETKAQAYV & 23 & 68 & 90 & 1.317 & 1.012 \\
\hline ALQDAGKSEDEVKEFETKAQAY & 22 & 68 & 89 & 2.36 & 0.995 \\
\hline ALQDAGKSEDEVKEFETKAQA & 21 & 68 & 88 & 2.007 & 0.987 \\
\hline ALQDAGKSEDEVKEFETKAQ & 20 & 68 & 87 & 2.59 & 0.983 \\
\hline ALQDAGKSEDEVKEFETKA & 19 & 68 & 86 & 1.984 & 0.982 \\
\hline ALQDAGKSEDEVKEFETK & 18 & 68 & 85 & 2.607 & 0.977 \\
\hline ALQDAGKSEDEVKEFET & 17 & 68 & 84 & 1.747 & 0.98 \\
\hline ALQDAGKSEDEVKEFE & 16 & 68 & 83 & 1.596 & 0.984 \\
\hline ALQDAGKSEDEVKEF & 15 & 68 & 82 & 1.218 & 0.993 \\
\hline ALQDAGKSEDEVKE & 14 & 68 & 81 & 1.864 & 0.986 \\
\hline ALQDAGKSEDEVK & 13 & 68 & 80 & 1.421 & 0.997 \\
\hline ALQDAGKSEDEV & 12 & 68 & 79 & 0.934 & 1.002 \\
\hline ALQDAGKSEDE & 11 & 68 & 78 & 1.684 & 0.968 \\
\hline ALQDAGKSED & 10 & 68 & 77 & 1.254 & 0.979 \\
\hline ALQDAGKSE & 9 & 68 & 76 & 0.975 & 0.992 \\
\hline ALQDAGKS & 8 & 68 & 75 & 0.738 & 1.009 \\
\hline ALQDAGK & 7 & 68 & 74 & 0.732 & 1.009 \\
\hline ALQDAG & 6 & 68 & 73 & 0.476 & 1.022 \\
\hline ALQDA & 5 & 68 & 72 & 0.621 & 1.052 \\
\hline ALQD & 4 & 68 & 71 & 0.802 & 1.049 \\
\hline ALQ & 3 & 68 & 70 & 0.627 & 1.11 \\
\hline LQDAGKSEDEVKEFETKAQAYV & 22 & 69 & 90 & 1.734 & 1.01 \\
\hline QDAGKSEDEVKEFETKAQAYV & 21 & 70 & 90 & 2.801 & 0.998 \\
\hline DAGKSEDEVKEFETKAQAYV & 20 & 71 & 90 & 2.109 & 0.997 \\
\hline AGKSEDEVKEFETKAQAYV & 19 & 72 & 90 & 1.676 & 1.004 \\
\hline GKSEDEVKEFETKAQAYV & 18 & 73 & 90 & 2.202 & 1.001 \\
\hline KSEDEVKEFETKAQAYV & 17 & 74 & 90 & 2.981 & 1.008 \\
\hline SEDEVKEFETKAQAYV & 16 & 75 & 90 & 1.966 & 1.013 \\
\hline EDEVKEFETKAQAYV & 15 & 76 & 90 & 1.938 & 1.013 \\
\hline DEVKEFETKAQAYV & 14 & 77 & 90 & 1.483 & 1.025 \\
\hline EVKEFETKAQAYV & 13 & 78 & 90 & 1.172 & 1.037 \\
\hline VKEFETKAQAYV & 12 & 79 & 90 & 0.89 & 1.053 \\
\hline KEFETKAQAYV & 11 & 80 & 90 & 1.605 & 1.023 \\
\hline EFETKAQAYV & 10 & 81 & 90 & 1.035 & 1.032 \\
\hline FETKAQAYV & 9 & 82 & 90 & 0.776 & 1.052 \\
\hline ETKAQAYV & 8 & 83 & 90 & 1.174 & 1.047 \\
\hline TKAQAYV & 7 & 84 & 90 & 0.901 & 1.075 \\
\hline KAQAYV & 6 & 85 & 90 & 0.812 & 1.103 \\
\hline AQAYV & 5 & 86 & 90 & 0.524 & 1.137 \\
\hline QAYV & 4 & 87 & 90 & 0.678 & 1.156 \\
\hline AYV & 3 & 88 & 90 & 0.51 & 1.203 \\
\hline FYTG & 4 & 103 & 106 & 0.645 & 1.009 \\
\hline
\end{tabular}




\begin{tabular}{cccccc}
\hline FYT & 3 & 103 & 105 & 0.851 & 1.054 \\
NPD & 3 & 110 & 112 & 1.804 & 0.902 \\
\hline
\end{tabular}

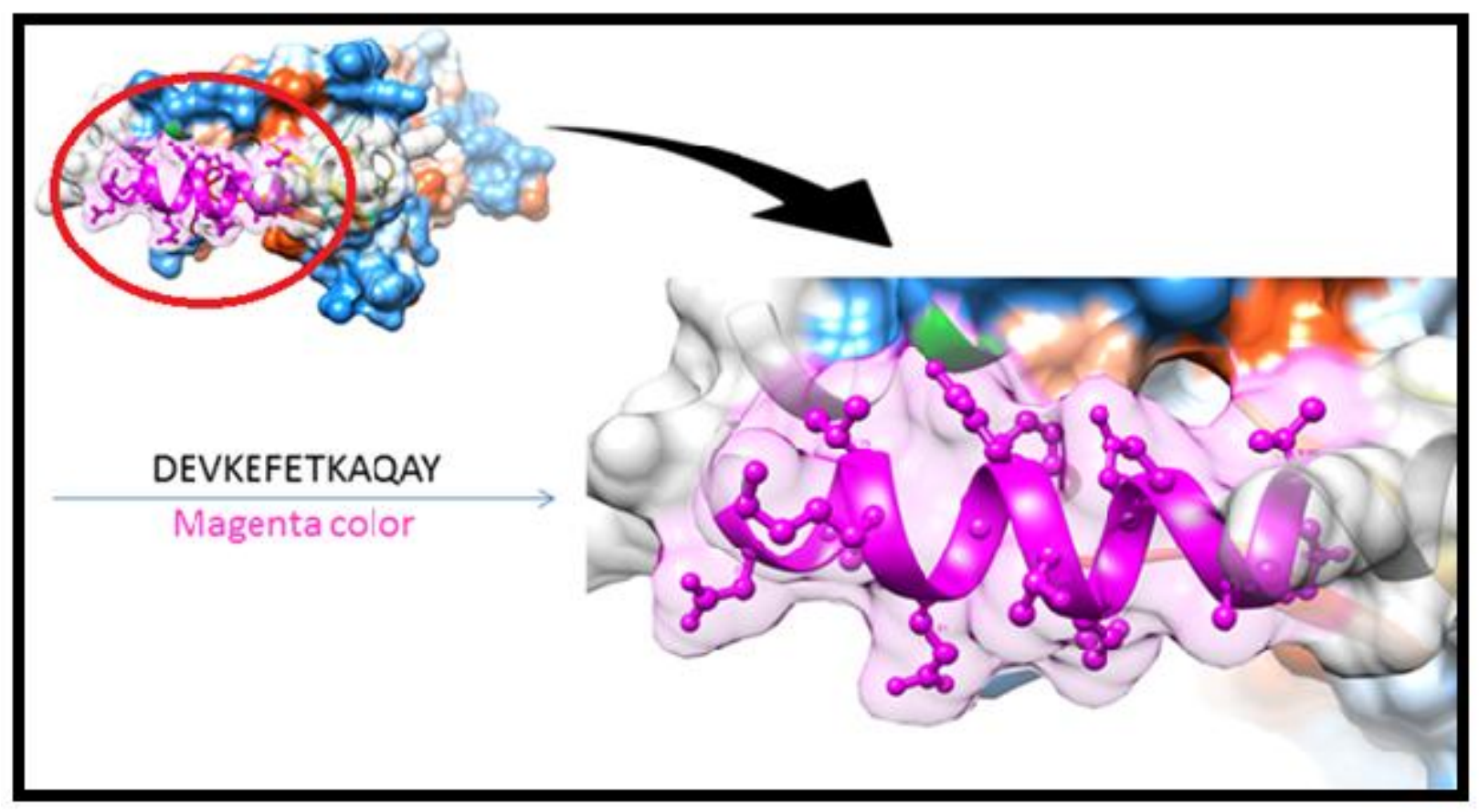

Fig 5 B cell epitopes proposed. the arrow show positions of (DEVKEFETKAQAY) with magenta color in structural level of Madurella. Mycetomatis TCTP.

Table2 The most Promising epitopesand their corresponding MHC-1 alleles

\section{Peptide}

YLKAYMKSV

TSFDKKSYL

YMKSVKKAL

\section{Alleles}

HLA-A*02:01,HLA-A*02:06,HLA-B*08:01,HLA-C*12:03,HLA-C*14:02

HLA-A*68:02,HLA-C*03:03,HLA-C*06:02,HLA-C*07:01,HLA-C*12:03,HLA-C*15:02

HLA-B*08:01,HLA-B*15:01,HLA-B*15:02,HLA-C*03:03,HLA-C*07:02,HLA-C*12:03,HLA-C*14:02 


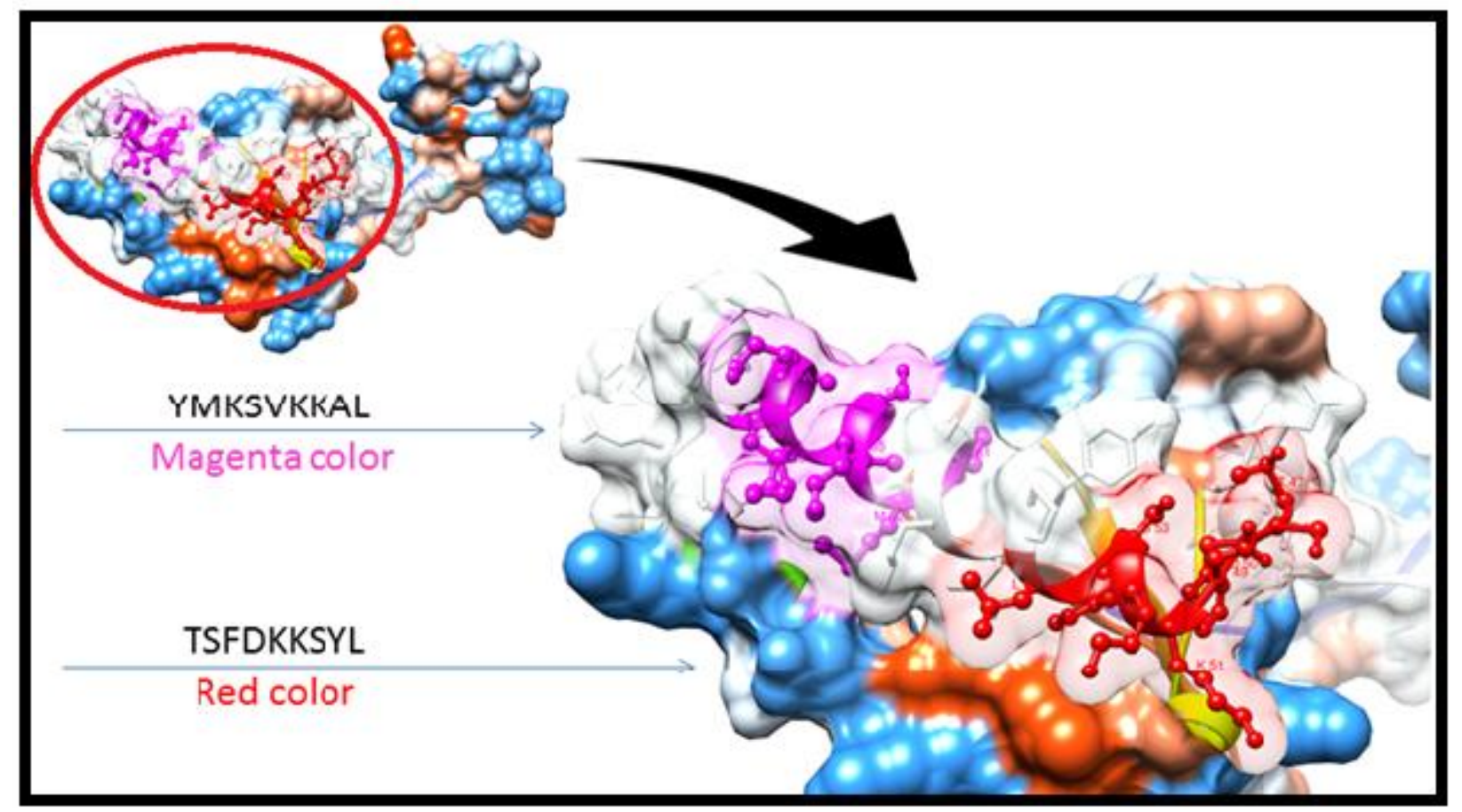

Fig $6 \mathrm{~T}$ cell epitopes proposed that interact with MHCI. The arrows show position of (YMKSVKKAL)with magenta color , (TSFDKKSYL) with red color in structural level of Madurella.Mycetomatis TCTP 


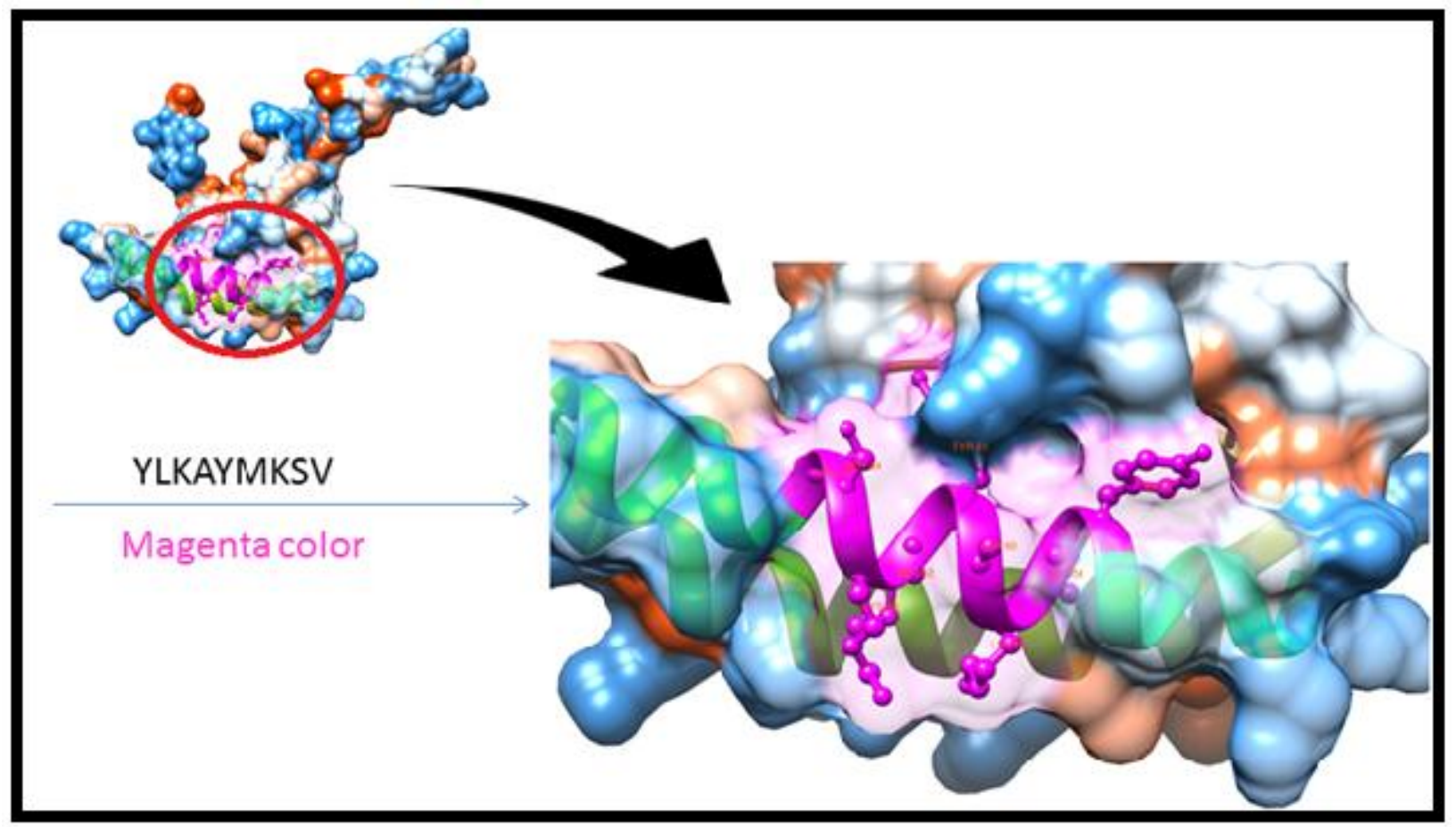

Fig $7 \mathrm{~T}$ cell epitopes proposed that interact with MHCI. The arrow show position of (YLKAYMKSV)with magenta color in structural level of Madurella Mycetomatis TCTP

Table 3 The most Promising epitopes and their corresponding MHC- II alleles

\begin{tabular}{|c|c|}
\hline Peptide & Alleles \\
\hline FRLQSTSFD & 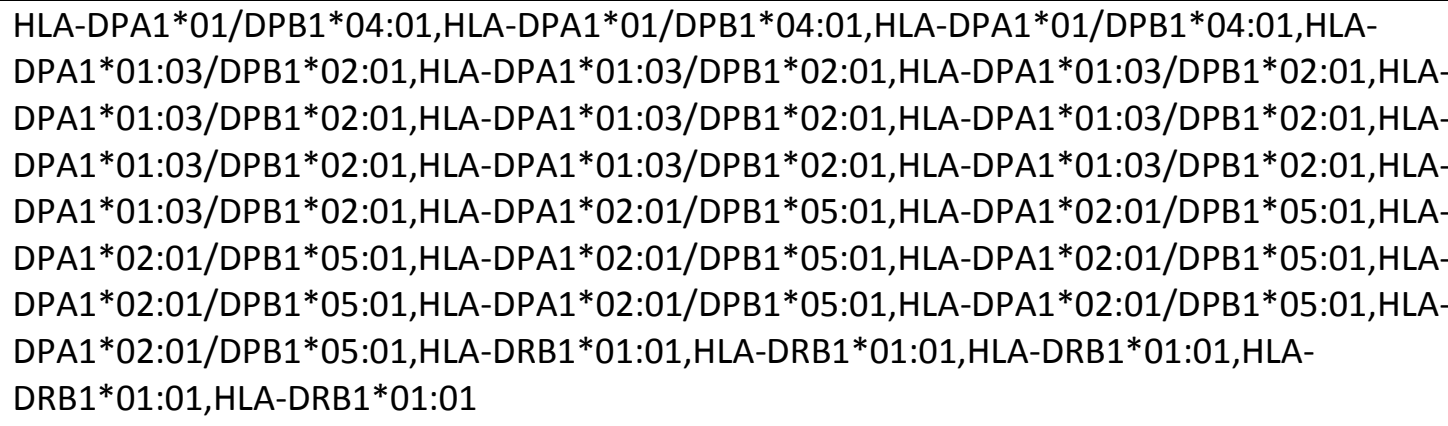 \\
\hline
\end{tabular}




\begin{tabular}{ll}
\hline YLKAYMKS & HLA-DRB1*11:01,HLA-DRB1*11:01,HLA-DRB1*11:01,HLA-DRB1*11:01,HLA-DRB1*11:01,HLA- \\
& DRB1*11:01,HLA-DRB1*11:01,HLA-DRB1*11:01,HLA-DRB1*11:01,HLA-DRB1*15:01,HLA- \\
& DRB1*15:01,HLA-DRB1*15:01,HLA-DRB1*15:01,HLA-DRB1*15:01,HLA-DRB1*15:01,HLA- \\
& DRB1*15:01,HLA-DRB1*15:01,HLA-DRB1*15:01
\end{tabular}

YLPYLKAYM HLA-DRB1*15:01,HLA-DRB3*01:01,HLA-DRB3*01:01,HLA-DRB3*01:01,HLA-DRB3*01:01,HLADRB5*01:01,HLA-DRB5*01:01,HLA-DRB5*01:01,HLA-DRB5*01:01,HLA-DRB5*01:01,HLADRB5*01:01,HLA-DRB5*01:01

LQSTSFDKK HLA-DRB1*04:05,HLA-DRB1*04:05,HLA-DRB1*07:01,HLA-DRB1*07:01,HLA-DRB1*07:01,HLADRB1*07:01,HLA-DRB1*07:01,HLA-DRB1*07:01,HLA-DRB1*07:01,HLA-DRB1*08:02

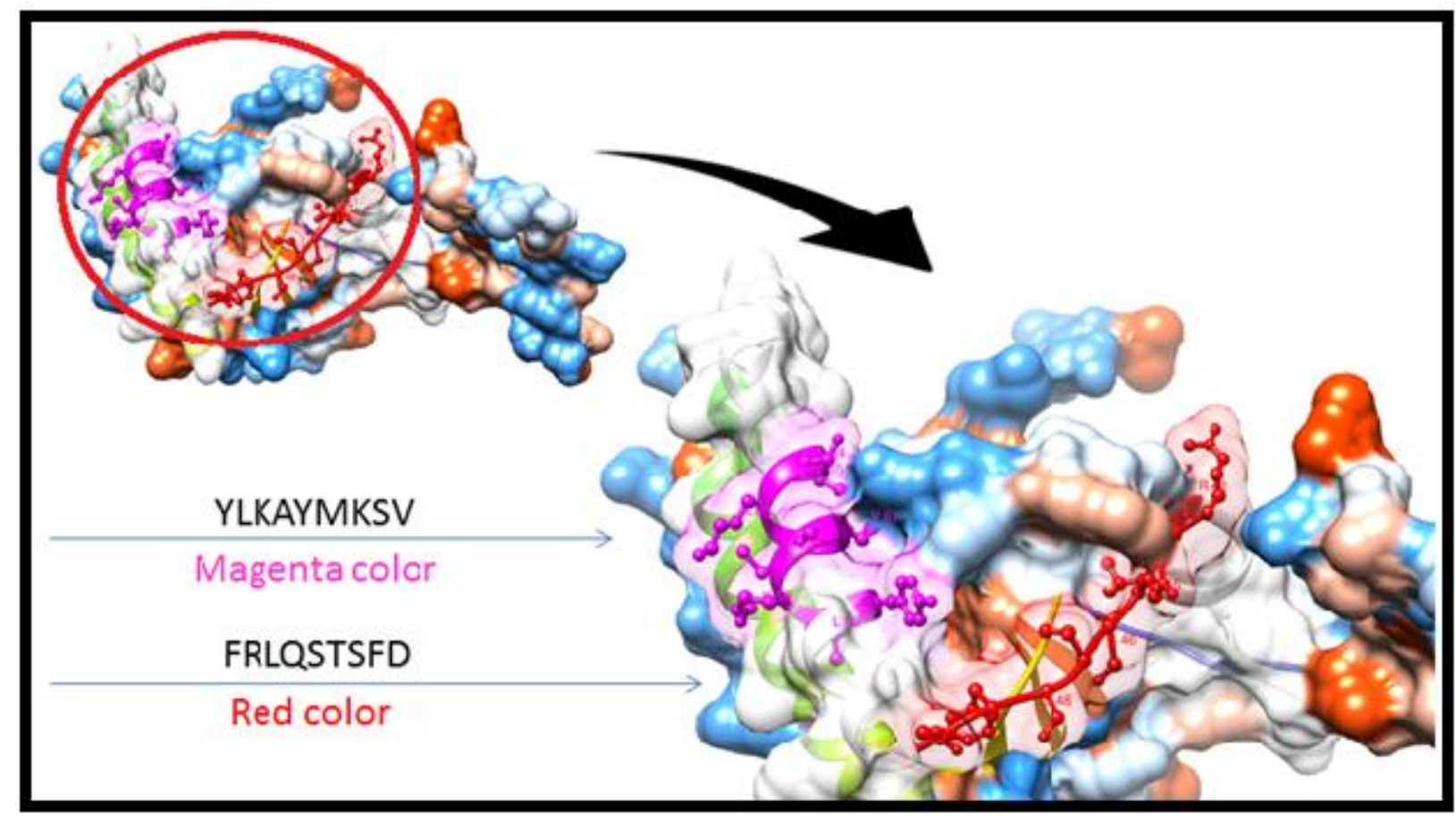

Fig $8 \mathrm{~T}$ cell epitopes proposed that interact with MHCII. The arrow show position of(YLKAYMKSV) with magenta color and (FRLQSTSFD) with red in structural level of glycoprotein of Madurella Mycetomatis TCTP 


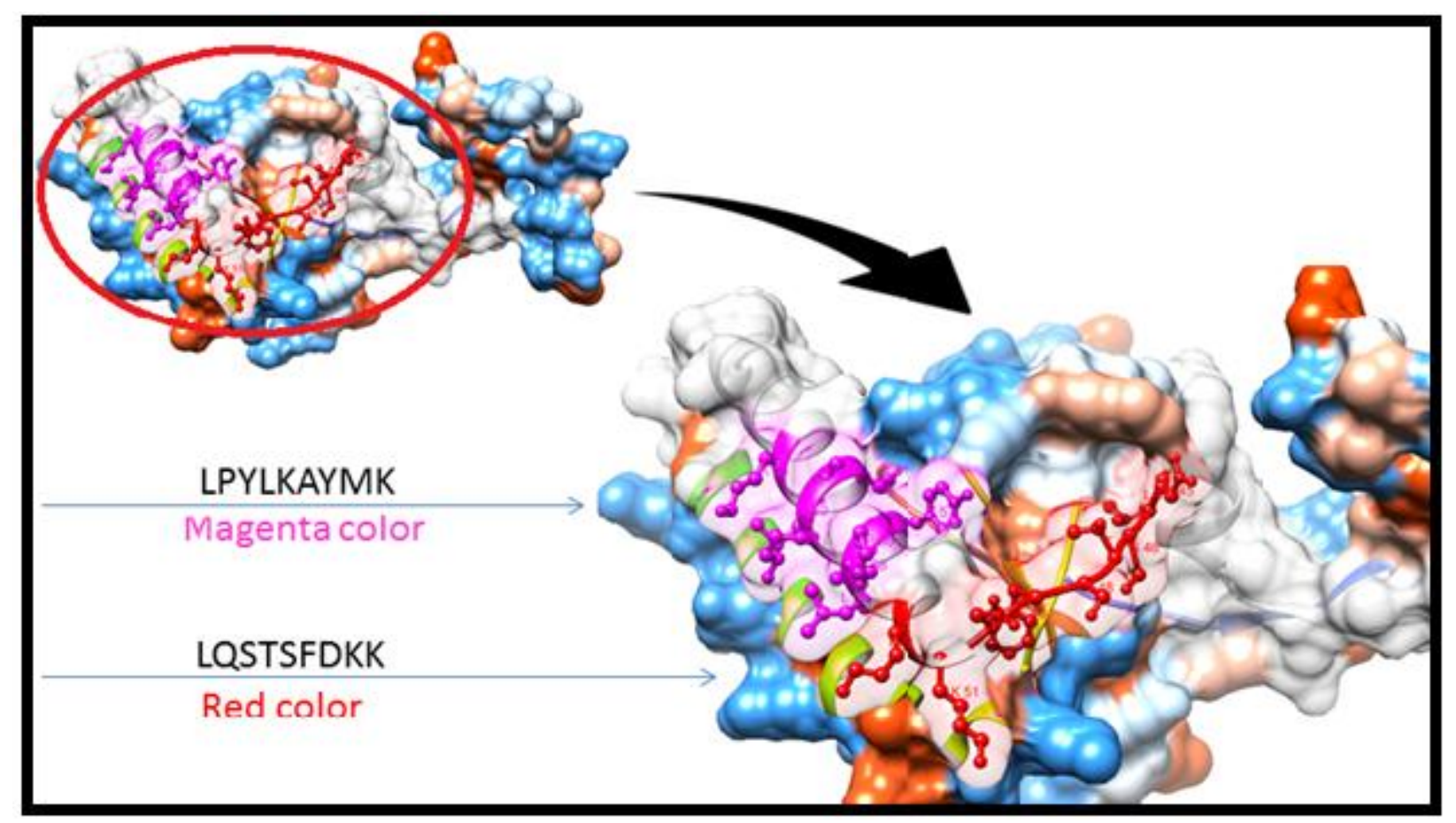

Fig $9 \mathrm{~T}$ cell epitopes proposed that interact with MHCII. The arrow show position of (LPYLKAYMK) with magenta and (LQSTSFDKK) with red color in structural level of 


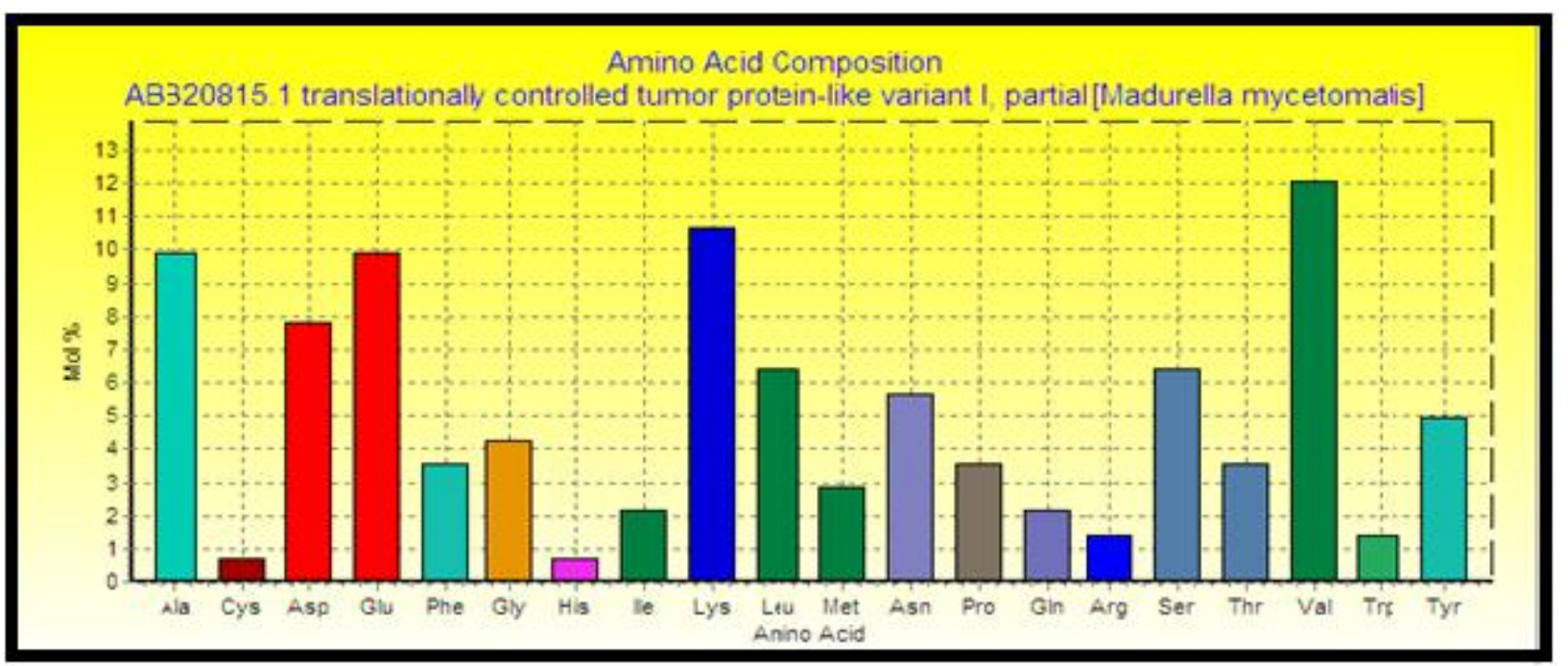

Fig 10 TCTP Protein analysis graph (Bioedit program, version 7.0.9.0)

Table 4 Atomic composition of Madurella mycetomatisTCTP.

\begin{tabular}{lcc}
\hline Elements & Symbol & No. of atoms \\
\hline Carbon & $\mathrm{C}$ & 705 \\
Hydrogen & $\mathrm{H}$ & 1095 \\
Nitrogen & $\mathrm{N}$ & 177 \\
Oxygen & $\mathrm{O}$ & 224 \\
Sulfur & $\mathrm{S}$ & 5 \\
\hline
\end{tabular}


Table 5 The population coverage of Whole world and Sudan for the most promising MHCI, MHCII, MHC I/ II, Shared epitope as MHCI,II epitopes and their alleles.

\section{Country MHCI MHCII MHCI,II Shared epitope as MHCI,II}

\section{Discussion:}

In our study, different peptide vaccines against TCTP of Madurella. mycetomatis are presented for the first time.

Design of effective vaccine is essential as protection tool against the disease because vaccines and effective treatments of mycetoma are not available till now (12). Peptide vaccines overcome the side effects of conventional vaccines.

TCTP is present in form of two alleles. Across the retrieved sequences of TCTP, the region from 42-93 is highly conserved. IEDB tools were used. Seven epitopes from the conserved regions were predicted eliciting B lymphocyte using Bepipred test, 37 epitopes were potentially in the surface by passing Emini surface accessability test while 15 epitopes were antigenic using

Kolaskar and Tongaonkar test. Only four epitopes passed the three tools (DEVKEFETKAQAYV, EVKEFETKAQAYV, EFETKAQAYV, ETKAQAYV).

111 epitopes were predicted to interact with MHCI alleles with IC50 < 500. Three of them were most promising (YLKAYMKSV, TSFDKKSYL, YMKSVKKAL). 274 predicted epitopes were interacted with MHCII alleles with IC50 < 100. Four of them were most promising (FRLQSTSFD, YLKAYMKSV, YLPYLKAYM, LQSTSFDKK).

Whole world, Sudan and Mexico had the highest population coverages concerning the promising peptides with high affinity to MHC class I alleles alone and the promising peptides with high affinity to MHC class I and class II alleles combined together. 
The epitope (YMKSVKKAL) was the most promising one concerning its binding with MHCI alleles, while (FRLQSTSFD) was the most promising for MHC II. The epitope (YLKAYMKSV) is shared between MHC I and II. For the population coverage of M. mycetomatis TCTP vaccine, Sudan $(90.39 \%)$ had the highest percentage for MHC I.

The whole world beside the mycetoma belt will benefit from this vaccine upon its successful development. TCTP is also present in many other species, so predicted peptides can be used as mulifactorial vaccine to protect from many diseases at the same time.

Many studies predicted peptide vaccines for different microorganisms such as, Rubella, Ebola, Dengue, Zika, Lagos rabies virus, HPV, HIV, HCV, malaria, anthrax, influenza, and swine fever using immunoinformatics tools (37-43). A published study found that the peptide FFKEHGVPL had a very strong binding affinity to MHCII alleles which made it a strong candidate as epitope based vaccine against Fructose bisphosphate aldolase of Madurella mycetomatis (44). Limitations were seen upon the calcutation of the population coverages concerning the promising peptides with MHC class II for (Venezuela, Senegal, Mexico, India, Colombia and Argentina), beside the combined MHC class I and class II for (Senegal, Mexico, India, Colombia, Argentina and Venezuela)due to missed alleles in the database.The peptide YLKAYMKSV is shared between MHC class I and class II but its percentage in the population coverage was not so high., Further invivo and invitro studies are recommended to prove the effectiveness of these peptides for vaccination.

\section{Conclusion:}

We conclude that due to the fact that Peptide vaccines overcome the side effects of conventional vaccines, our study presented for the first time different peptide vaccines against TCTP of Madurella. Mycetomatis. The epitope (YMKSVKKAL) was the most promising one concerning its binding with MHCI alleles, while (FRLQSTSFD) was the most promising for MHC II. The epitope (YLKAYMKSV) is shared betweenMHC I and II. For the population coverage of $M$. Mycetomatis TCTP vaccine Sudan (90.39\%) had the highest percentage for MHC I. 


\section{Acknowledgment}

Deep thanks to staff of Africa City of Technology. Pure thanks to everyone who supported us to conduct this paper.

\section{References:}

1. Bonifaz A, Tirado-Sanchez A, Calderon L, Saul A, Araiza J, Hernandez M, et al. Mycetoma: experience of 482 cases in a single center in Mexico. PLoS neglected tropical diseases. 2014;8(8):e3102. 2. Brufman T, Ben-Ami R, Mizrahi M, Bash E, Paran Y. Mycetoma of the Foot Caused by Madurella Mycetomatis in Immigrants from Sudan. The Israel Medical Association journal : IMAJ. 2015;17(7):41820.

3. Mahgoub E. Mycetoma Foot. JSM. 2017;2(2):1023.

4. Zijlstra EE, van de Sande WWJ, Welsh O, Mahgoub ES, Goodfellow M, Fahal AH. Mycetoma: a unique neglected tropical disease. The Lancet Infectious diseases. 2016;16(1):100-12.

5. Develoux M. [Mycetoma and their treatment]. Journal de mycologie medicale. 2016;26(2):7785. Les mycetomes et leur traitement.

6. Shrivastava SR, Shrivastava PS, Ramasamy J. The public health implications of mycetoma: A neglected tropical disease. CHRISMED Journal of Health and Research. 2017;4(4):289.

7. Bitan O, Wiener-Well Y, Segal R, Schwartz E. Mycetoma (Madura Foot) in Israel: Recent Cases and a Systematic Review of the Literature. The American journal of tropical medicine and hygiene. 2017;96(6):1355-61.

8. Elfadil H, Fahal A, Kloezen W, Ahmed EM, van de Sande W. The in vitro antifungal activity of sudanese medicinal plants against Madurella mycetomatis, the eumycetoma major causative agent. PLoS neglected tropical diseases. 2015;9(3):e0003488.

9. Relhan V, Mahajan K, Agarwal P, Garg VK. Mycetoma: An Update. Indian journal of dermatology. 2017;62(4):332-40.

10. Efared B, Tahiri L, Boubacar MS, Atsam-Ebang G, Hammas N, Chbani L. Mycetoma in a nonendemic area: a diagnostic challenge. BMC clinical pathology. 2017;17(1):1.

11. Relhan V, Mahajan K, Agarwal P, Garg VK. Mycetoma: An Update. Indian journal of dermatology. 2017;62(4):332.

12. van de Sande WW, Fahal AH, Goodfellow M, Welsh O, Zijlstra E. The mycetoma knowledge gap: identification of research priorities. PLoS neglected tropical diseases. 2014;8(3):e2667.

13. Smit S, Derks MF, Bervoets S, Fahal A, van Leeuwen W, van Belkum A, et al. Genome Sequence of Madurella mycetomatis mm55, Isolated from a Human Mycetoma Case in Sudan. Genome announcements. 2016;4(3).

14. Ahmed SA, van de Sande WW, Desnos-Ollivier M, Fahal AH, Mhmoud NA, de Hoog GS. Application of Isothermal Amplification Techniques for Identification of Madurella mycetomatis, the Prevalent Agent of Human Mycetoma. Journal of clinical microbiology. 2015;53(10):3280-5.

15. Fahal A, Mahgoub el S, El Hassan AM, Abdel-Rahman ME. Mycetoma in the Sudan: an update from the Mycetoma Research Centre, University of Khartoum, Sudan. PLoS neglected tropical diseases. 2015;9(3):e0003679.

16. Nasr A, Abushouk A, Hamza A, Siddig E, Fahal AH. Th-1, Th-2 Cytokines Profile among Madurella mycetomatis Eumycetoma Patients. PLoS neglected tropical diseases. 2016;10(7):e0004862. 
17. van de Sande WW, Janse DJ, Hira V, Goedhart H, van der Zee R, Ahmed AO, et al. Translationally controlled tumor protein from Madurella mycetomatis, a marker for tumorous mycetoma progression. Journal of immunology. 2006;177(3):1997-2005.

18. Ahmed AA, van de Sande W, Fahal AH. Mycetoma laboratory diagnosis: Review article. PLoS neglected tropical diseases. 2017;11(8):e0005638.

19. Armstrong-James D, Brown GD, Netea MG, Zelante T, Gresnigt MS, van de Veerdonk FL, et al. Immunotherapeutic approaches to treatment of fungal diseases. The Lancet Infectious Diseases. 2017. 20. Acunzo J, Baylot V, So A, Rocchi P. TCTP as therapeutic target in cancers. Cancer treatment reviews. 2014;40(6):760-9.

21. Arcuri F, Papa S, Carducci A, Romagnoli R, Liberatori S, Riparbelli MG, et al. Translationally controlled tumor protein (TCTP) in the human prostate and prostate cancer cells: expression, distribution, and calcium binding activity. The Prostate. 2004;60(2):130-40.

22. de Klerk N, de Vogel C, Fahal A, van Belkum A, van de Sande WW. Fructose-bisphosphate aldolase and pyruvate kinase, two novel immunogens in Madurella mycetomatis. Sabouraudia. 2012;50(2):143-51.

23. Hall TA, editor. BioEdit: a user-friendly biological sequence alignment editor and analysis program for Windows 95/98/NT. Nucleic acids symposium series; 1999: [London]: Information Retrieval Ltd., c1979-c2000.

24. Bonifaz A, Tirado-Sánchez A, Calderón L, Saúl A, Araiza J, Hernández M, et al. Mycetoma: experience of 482 cases in a single center in Mexico. PLoS neglected tropical diseases. 2014;8(8):e3102. 25. Larsen JE, Lund O, Nielsen M. Improved method for predicting linear B-cell epitopes. Immunome research. 2006;2(1):2.

26. Emini EA, Hughes JV, Perlow D, Boger J. Induction of hepatitis A virus-neutralizing antibody by a virus-specific synthetic peptide. Journal of virology. 1985;55(3):836-9.

27. Kolaskar A, Tongaonkar PC. A semi-empirical method for prediction of antigenic determinants on protein antigens. FEBS letters. 1990;276(1-2):172-4.

28. Andreatta $M$, Nielsen M. Gapped sequence alignment using artificial neural networks: application to the MHC class I system. Bioinformatics. 2015;32(4):511-7.

29. Buus S, Lauemøller S, Worning P, Kesmir C, Frimurer T, Corbet S, et al. Sensitive quantitative predictions of peptide-MHC binding by a 'Query by Committee'artificial neural network approach. HLA. 2003;62(5):378-84.

30. Nielsen M, Lundegaard C, Worning P, Lauemøller SL, Lamberth K, Buus S, et al. Reliable prediction of T-cell epitopes using neural networks with novel sequence representations. Protein Science. 2003;12(5):1007-17.

31. Peters B, Sette A. Generating quantitative models describing the sequence specificity of biological processes with the stabilized matrix method. BMC bioinformatics. 2005;6(1):132.

32. Lundegaard C, Lamberth K, Harndahl M, Buus S, Lund O, Nielsen M. NetMHC-3.0: accurate web accessible predictions of human, mouse and monkey MHC class I affinities for peptides of length 8-11. Nucleic acids research. 2008;36(suppl_2):W509-W12.

33. Wang P, Sidney J, Dow C, Mothe B, Sette A, Peters B. A systematic assessment of MHC class II peptide binding predictions and evaluation of a consensus approach. PLoS computational biology. 2008;4(4):e1000048.

34. Wang P, Sidney J, Kim Y, Sette A, Lund O, Nielsen M, et al. Peptide binding predictions for HLA DR, DP and DQ molecules. BMC bioinformatics. 2010;11(1):568.

35. Zhang $Q$, Wang $P$, Kim $Y$, Haste-Andersen $P$, Beaver J, Bourne PE, et al. Immune epitope database analysis resource (IEDB-AR). Nucleic acids research. 2008;36(suppl_2):W513-W8.

36. Bui H-H, Sidney J, Dinh K, Southwood S, Newman MJ, Sette A. Predicting population coverage of T-cell epitope-based diagnostics and vaccines. BMC bioinformatics. 2006;7(1):153. 
37. Ahmed O, Abdelhalim A, Obi S, Ali K, Hamdi A. Immunoinformati c Approach for Epitope-Based Peptide Vaccine against Lagos Rabies Viru s Glycoprotein G. Immunome Res. 2017;13(137):2.

38. Abu-haraz AH, Abd-elrahman KA, Ibrahim MS, Hussien WH, Mohammed MS, Badawi MM, et al. Multi epitope peptide vaccine prediction against Sudan Ebola virus using Immuno-informatics approaches. Advanced Techniques in Biology \& Medicin e. 2017;5:1-22.

39. Badawi MM, Alla AAF, Alam SS, Mohamed WA, Osman DAN-E, Ali SAAA, et al. Immunoinformatics Predication and in silico Modeling of Epitope-Based Peptide Vaccine Against virulent Newcastle Disease Viruses. Am J Infectious Dis Microbiol. 2016;4(3):61-71.

40. Mohammed A, Hashim O, Elrahman K, Hamdi A, Hassan M. Epitope-Based Peptide Vaccine Design Against Mokola Rabies Virus Glycoprotein G Utilizing In Silico Approaches. Immunome Res. 2017;13(144):2.

41. Albagi S, Ahmed O, Gumaa M, Abd_elrahman K, Abu-Haraz A. Immunoinformatics-Peptide Driven Vaccine and In silico Modeling for Duvenhage Rabies Virus Glycoprotein G. J Clin Cell Immunol. 2017;8(517):2.

42. Badawi MM, Osman MM, Alla A, Ahmedani AM, hamed Abdalla M, Gasemelseed MM, et al. Highly conserved epitopes of Zika envelope glycoprotein may act as a novel peptide vaccine with high coverage: immunoinformatics approach. Am J Biomed Res. 2016;4:46-60.

43. Elgenaid S, Al-Hajj E, Ibrahim A, Essa M, Abu-haraz A. Prediction of Multiple Peptide Based Vaccine from E1, E2 and Capsid Proteins of Rubella Virus: An In-Silico Approach. Immunome Res. 2018;14(146):2.

44. Mohammed AA, ALnaby AM, Sabeel SM, AbdElmarouf FM, Dirar Al, Ali MM, et al. Epitopebased peptide vaccine against Fructose-bisphosphate aldolase (FBA) of Madurella mycetomatis using immunoinformatics approaches. bioRxiv. 2018:352625. 